\title{
ANÁLISIS DEL RIESGO DE INUNDACIÓN EN MOTILLA DEL PALANCAR (CUENCA, ESPAÑA)
}

\author{
Alberto Martínez Escribano \\ Máster en Planificación y Gestión de Riesgos Naturales \\ Universidad de Alicante
}

\section{RESUMEN}

La localidad de Motilla del Palancar (Cuenca), situada en el interior este peninsular, se encuentra expuesta a las inundaciones causadas por dos cursos de agua. Uno de ellos es el río Valdemembra, con una cuenca extensa y longitudinal, pero con poco caudal. La otra es una cuenca pequeña, redondeada y sin un cauce definido que desagua hacia el río a través de la calle principal de la localidad y que llamaremos Riato. Ambas provocan inundaciones: el río menos frecuentes, con alturas de agua importantes, y que han llegado a causar víctimas; en el Riato, más frecuentes pero con menos altura de lámina de agua. La alta exposición debida a la deficiente ordenación territorial junto a una elevada vulnerabilidad se suman a una peligrosidad moderada para desembocar en un escenario de riesgo claro tanto para la población como para los bienes expuestos, agravado por los cambios de usos del suelo dentro de las cuencas vertientes.

Palabras clave: riesgo de inundación, Motilla del Palancar, exposición, vulnerabilidad, peligrosidad, ordenación territorial, usos del suelo

\section{ABSTRACT}

\section{Flood Risk Analisis in Motilla del Palancar (Cuenca, Spain)}

The village of Motilla del Palancar (Cuenca), placed in the inside east of the Iberian Peninsula, is exposed to floods caused by two watercourses. One of them it's the Valdemembra River, with a wide and lengthwise basin, but with a low flow. The other one is a small, rounded and without a defined channel basin that drains into the river through the village's main street we will call Riato. Both generate floods: the river less frequently, with significant water height, and that have even caused deaths; the Riato, more frequently but with low water height. The high exposure due to the territorial poor management with a high vulnerability added to a moderate hazard generate a clear risk scene both for people and properties exposed, aggravated by land uses changes inside catchment areas.

Key words: flood risk, Motilla del Palancar, exposure, vulnerability, hazard, territorial management, land uses.

\section{INTRODUCCIÓN}

Las inundaciones son el riesgo natural que más víctimas y daños causa en España. A lo largo del siglo XX ha habido en España grandes catástrofes que han dejado cientos de víctimas debidas a inundaciones. Ejemplos trágicos de estos sucesos son las inundaciones en Barcelona en 1962 con más de 600 muertos o la 'gota fría' que en 1973 mató a más de 300 personas en Murcia y Almería, concentradas en la costa mediterránea principalmente. Desde los años 70 el número de fallecidos ha ido disminuyendo. Sin embargo, en los años 90 aún se produjeron desastres como el de Biescas o el de Badajoz, con decenas de muertos. La mayoría de los últimos desastres se han debido a lluvias torrenciales y a crecidas 'relámpago'

Contacto: albertome20@gmail.com 
en cuencas pequeñas del interior alejadas de la costa mediterránea. En las cuencas de los grandes ríos prácticamente no hay víctimas desde que los medios de comunicación permiten avisar a las poblaciones aguas abajo de la llegada de la crecida. En los últimos años las víctimas originadas por inundaciones son muertes aisladas o en grupos pequeños, inferiores a 5. Generalmente estas muertes son personas arrastradas cuando caminan por la calle o intentan cruzar un paso inundable con el coche. Por otro lado, las pérdidas económicas han aumentado junto con el desarrollo económico del país y el crecimiento de la población. Los núcleos urbanos han crecido mucho y han ocupado zonas inundables, exponiendo bienes y personas a las inundaciones.

Fig. 1. Localización de la zona de estudio.

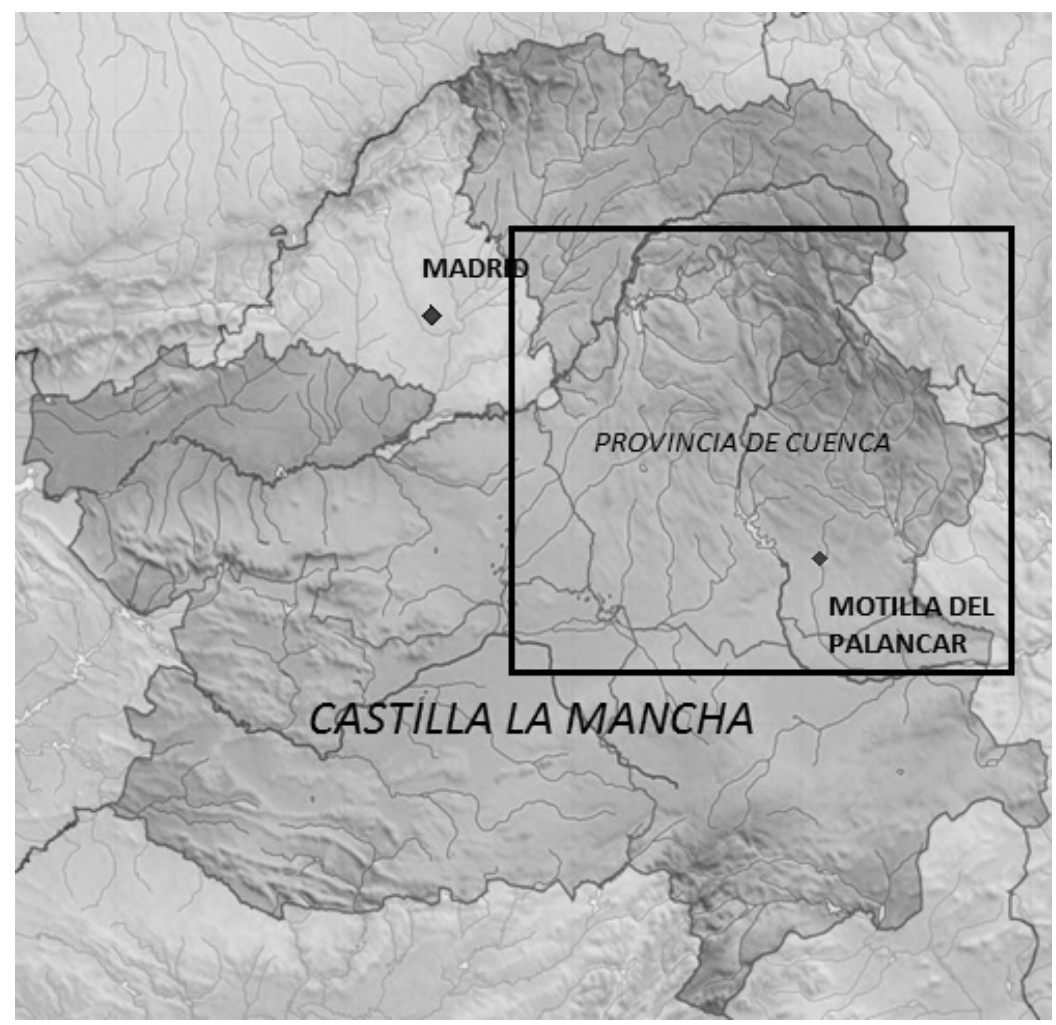

Mientras que en las comunidades del litoral mediterráneo se han desarrollado planes de prevención del riesgo de inundación desde los años 80 , reduciéndose mucho las pérdidas de vidas humanas, las comunidades del interior han recibido menos atención. La Comunidad Valenciana, la Región de Murcia o Cataluña poseen planes de acción territorial más o menos completos. Por el contrario, las comunidades del interior no poseen ni siquiera cartografía detallada de su territorio y menos aún de riesgo de inundación. Así, los últimos grandes desastres durante los años 90 han ocurrido en comunidades del interior, lejos de la costa, como el desastre de Biescas en Huesca, el Cerro de los Reyes en Badajoz o Yebra y Almoguera en Guadalajara. Respecto a Castilla-La Mancha históricamente ha padecido inundaciones que han provocado importantes desastres. La inundación de Consuegra el 11 de septiembre de 1891 que dejó 354 fallecidos o la inundación de Valdepeñas en 1979 con 21 fallecidos, sin olvidar la de Yebra y Almoguera que dejó 10 muertos en 1995, son ejemplos de ello.

En la propia localidad de Motilla del Palancar las inundaciones forman parte de su historia. Tal es así, que algunos autores afirman que el nombre proviene de una mota construida para protegerse de las avenidas de la cañada junto a la que se asentaba. La inundación más antigua que se conoce es de principios del siglo XIX. Pero la peor de todas ocurrió en 1921, cuando una fuerte tormenta generó una gran avenida del río Valdemembra que inundó con hasta 2 metros de agua la zona baja del pueblo, destruyendo decenas de casas y matando a 12 vecinos. 


\section{OBJETIVOS}

El principal objetivo del trabajo es llevar a cabo un diagnóstico de la situación actual del riesgo de inundación en el casco urbano de la localidad. Anteriormente la localidad no contaba con ningún estudio sobre riesgos de ningún tipo, y tampoco sobre riesgo de inundación, a pesar de haber sufrido históricamente inundaciones graves que han dejado víctimas, como la de septiembre de 1921. Tan solo había un estudio de inundabilidad de la antigua Confederación Hidrográfica del Júcar (CHJ), actual Demarcación Hidrográfica del Júcar (DHJ). En dicho estudio se calculaba el caudal máximo esperable para los dos cauces, pero sin marcar zonas inundables. Se pretende realizar una cartografía detallada del riesgo para diferentes periodos de retorno a partir de los datos históricos y las adaptaciones de la construcción a las inundaciones. Así mismo se recopiló la cartografía disponible sobre riesgo de inundación de la zona, comparándola con la obtenida y realizando un análisis de las diferencias y las posibles causas de dichas diferencias. Por último se recogen una serie de recomendaciones de actuaciones a llevar a cabo para minimizar el riesgo de inundación en la localidad.

\section{METODOLOGÍA}

La metodología empleada podríamos dividirla en dos procesos diferenciados: por un lado, y en primer lugar, se realizó una recopilación de información que pudiese ser útil en el estudio, y por otro lado, se realizó el diagnóstico del riesgo a partir de dicha información.

\subsection{Episodios de inundación y origen sinóptico de los mismos}

En la primera fase y como primer paso se recopiló información en diferentes fuentes sobre inundaciones históricas en la localidad. Se recurrió a la consulta tanto de hemerotecas digitales, informes de la Confederación Hidrográfica del Júcar, estudios que poseía el ayuntamiento, webs dedicadas a la localidad y entrevistas con vecinos de la zona.

A continuación se analizó las situaciones sinópticas que originaron las precipitaciones causantes de las inundaciones. Para ello se consultaron los mapas sinópticos a $850 \mathrm{hPa}$ y a $500 \mathrm{hPa}$ disponibles en la web www.wetterzentrale.com. En la Tabla 1 se pueden consultar los episodios y las situaciones sinópticas recopiladas. Desde un punto de vista temporal, se puede observar que la mayor parte de los eventos se concentran a finales del verano, en especial finales de agosto y sobre todo en el mes de septiembre. Tan solo se recogen dos episodios fuera de esas fechas, uno en junio y otro en enero. El de junio responde a una tormenta generada por una onda corta de aire polar marítimo, mientras que la de enero de 1997 se debió a la fusión de la nieve caída los días previos. Por tanto, con los datos disponibles, la época del año con mayor probabilidad de ocurrencia de inundaciones en la localidad va desde mediados de agosto hasta finales de septiembre. Hay que resaltar que a principios de septiembre se celebra la feria y las fiestas patronales, instalándose la feria y la carpa del baile en la Avenida del Riato, lo que supone una instalación muy vulnerable localizada en el cauce de una rambla justo en las fechas más proclives a la ocurrencia de inundaciones. En la crecida de 2004 ya sufrieron daños importantes, suspendiéndose la feria y teniéndose que evacuar el recinto donde se celebraba el baile al verse inundado.

Desde un punto de vista sinóptico, los episodios de finales de verano se relacionan con vaguadas de aire polar marítimo que pueden estrangularse hasta formar una DANA en altura. Ambas situaciones generan una gran inestabilidad, ya que el aire frío en altura contrasta con las altas temperaturas de la superficie a finales de verano. En el caso de formación de una DANA la divergencia en capas altas favorece el crecimiento de grandes cumulonimbos que provocan tormentas muy intensas. Si en superficie hay viento procedente del sur o del este, aire cálido y muy húmedo procedente del Mediterráneo, se crea una gran inestabilidad en la atmósfera que desemboca en la formación de tormentas fuertes que pueden descargar importantes cantidades de lluvia en poco tiempo. 
Tablal. Episodios de inundación y su origen sinóptico:

\begin{tabular}{|c|c|c|}
\hline FECHA & DESCRIPCIÓN & SITUACIÓN ATMOSFÉRICA \\
\hline $1811-1812$ & Inundación de la localidad provoca una víctima & \\
\hline 21-09-1921 & $\begin{array}{l}\text { El río Valdemembra inunda la zona baja de la } \\
\text { localidad, destruyendo decenas de casas, causando } \\
12 \text { fallecidos y grandes daños económicos }\end{array}$ & $\begin{array}{l}\text { DANA al suroeste de la península Ibérica. Sector de } \\
\text { divergencia de vientos en altitud sobre la mitad este } \\
\text { peninsular. Entrada de vientos del norte de África en } \\
\text { los niveles bajos de la troposfera }\end{array}$ \\
\hline $17-09-1963$ & & $\begin{array}{l}\text { Vaguada de aire polar marítimo centrada en la } \\
\text { península Ibérica. Circulación de vientos indefinida } \\
\text { en superficie. Tormentas }\end{array}$ \\
\hline $31-08-1968$ & & $\begin{array}{l}\text { Vaguada de aire polar marítimo situada al oeste } \\
\text { de la península Ibérica. Circulación ciclónica en } \\
\text { superficie. }\end{array}$ \\
\hline 08 al 12-09-1996 & & $\begin{array}{l}\text { Vaguada y DANA formada dentro de ella al suroeste } \\
\text { de la península Ibérica. Circulación del segundo } \\
\text { cuadrante en superficie. }\end{array}$ \\
\hline 07-01-1997 & $\begin{array}{l}\text { Inundación de la vega del río. Probablemente } \\
\text { también de la zona baja de la población. }\end{array}$ & $\begin{array}{l}\text { Vaguada de aire polar marítimo centrada en } \\
\text { la península Ibérica. Circulación ciclónica en } \\
\text { superficie. }\end{array}$ \\
\hline $13-06-2004$ & $\begin{array}{l}\text { Fuerte tormenta que causa daños en la comarca. } \\
\text { Avenida en el Riato. }\end{array}$ & $\begin{array}{l}\text { Onda corta de aire polar sobre la península Ibérica. } \\
\text { Entrada de vientos del norte de África en superficie. } \\
\text { Tormentas. }\end{array}$ \\
\hline 31-08 al 05-09-2004 & $\begin{array}{l}\text { Varias tormentas provocan la inundación reiterada } \\
\text { de la avenida del Riato durante la feria, provocando } \\
\text { la suspensión de ésta y daños en las instalaciones. } \\
\text { Los días } 2 \text { y } 3 \text { el agua inunda la zona baja de la } \\
\text { localidad. }\end{array}$ & $\begin{array}{l}\text { Vaguada profunda de aire polar al oeste de la } \\
\text { península Ibérica. Fuerte difluencia en capas altas. } \\
\text { Inestabilidad elevada. Tormentas intensas. }\end{array}$ \\
\hline
\end{tabular}

\subsection{Diagnóstico del riesgo}

\subsubsection{Estudio de las cuencas vertientes}

En primer lugar se procedió a la realización de un estudio lo más detallado posible del medio físico de la cuenca y de la localidad. A continuación se estudiaron las adaptaciones de la construcción a las inundaciones y se desarrolló un mapa con la extensión esperable de las inundaciones y su calado para el río Valdemembra y para el Riato. Para ello se realizaron un estudio geológico, un estudio geomorfológico, un estudio climático, un estudio hidrológico y un estudio de los usos del suelo.

La cuenca del río Valdemembra aguas arriba de la localidad, desde un aspecto geológico, se caracteriza por la presencia de terrenos calcáreos mesozoicos formando relieves en la parte alta y media, estando el río encajado en ellos en la mayor parte de este tramo. El resto de la cuenca está formada por materiales detríticos terciarios, principalmente arcillas y areniscas. Cuando el río sale de los materiales calcáreos se adentra en una vega amplia sobre la que se asienta en parte la localidad de Motilla. Esta vega está rellena de materiales aluviales cuaternarios aportados por el río asentados sobre materiales detríticos del Terciario.

Geomorfológicamente la cuenca es alargada, con orientación norte sur (figura 2). La cuenca alta tiene una orientación noroeste-sureste, siguiendo la orientación de los relieves ibéricos, y se localiza entre los términos de Solera de Gabaldón y Almodóvar del Pinar. Está formada por un amplio valle asentado sobre un sinclinal que recoge el agua de los relieves calcáreos que forman los flancos del pliegue. La cuenca media se caracteriza por el cambio de dirección a norte-sur y por el encajamiento del río en una superficie de erosión de materiales calcáreos mesozoicos. La cuenca baja ocupa una amplia vega cultivada sobre la que se asienta la localidad en la margen derecha. A su paso por la localidad el río está encauzado. Por 
la margen izquierda los afluentes son muy cortos al estar muy próxima la divisoria con la cuenca de la Rambla de Albengibre. Los principales afluentes proceden de la margen derecha. De ellos, el principal es el Vallejo de Ana María, en el término de Gabaldón. Su cuenca se extiende por materiales arcillosos y areniscas, considerablemente impermeables y deleznables.

El clima de la zona se caracteriza por ser un clima mediterráneo continentalizado, con inviernos muy fríos y secos, veranos cálidos y secos, algo más frescos en la zona alta próxima a la Serranía, y precipitaciones concentradas en otoño y primavera. Las precipitaciones varían entre 400 y $600 \mathrm{~mm}$ al año, disminuyendo hacia el sur. Las nevadas son frecuentes en invierno, así como las heladas. Las lluvias suelen proceder de frentes atlánticos que barren la península de oeste a este, siendo mayores las precipitaciones cuando éstos entran del suroeste. Sin embargo, las mayores intensidades se dan durante las tormentas que afectan a la zona en verano, especialmente a finales de dicha estación.

La hidrogeología de la cuenca se caracteriza por asentarse sobre un sustrato principalmente calcáreo. Esto unido a la escasa pendiente general de la zona favorece la karstificación y la infiltración del agua superficial en el subsuelo. La cuenca se asienta sobre dos acuíferos importantes: uno al norte formado por los relieves calcáreos de la Serranía Conquense y otro el resto de la cuenca, el conocido acuífero 18 de La Mancha-Oriental. Este acuífero se encuentra sobre-explotado y los niveles piezométricos de la zona han bajado considerablemente. Así mismo, la presencia del río Júcar y el Cabriel muy encajados favorece el drenaje de los acuíferos de forma natural. Todo esto facilita que el agua desaparezca de superficie. En la cuenca alta sí hay fuentes y manantiales relacionados con el acuífero de la Serranía, y el río lleva agua hasta que sale de la vega de Almodóvar. En el resto del cauce hasta Motilla es habitual que el río esté seco, especialmente en verano, comportándose más como una rambla que como un río. De hecho en Motilla se le conoce como 'la rambla'. El resto de afluentes son arroyos o ramblas que no llevan agua prácticamente nunca. Así, el río presenta un caudal discontinuo, constante solo en periodos húmedos y durante el invierno, estando seco casi todos los veranos e incluso los inviernos de periodos de sequía. Por tanto, aguas arriba de Motilla, el río Valdemembra se comporta más como un río-rambla que como un río propiamente dicho.

Figura 2: Modelo digital del terreno de las cuencas afluentes a Motilla.

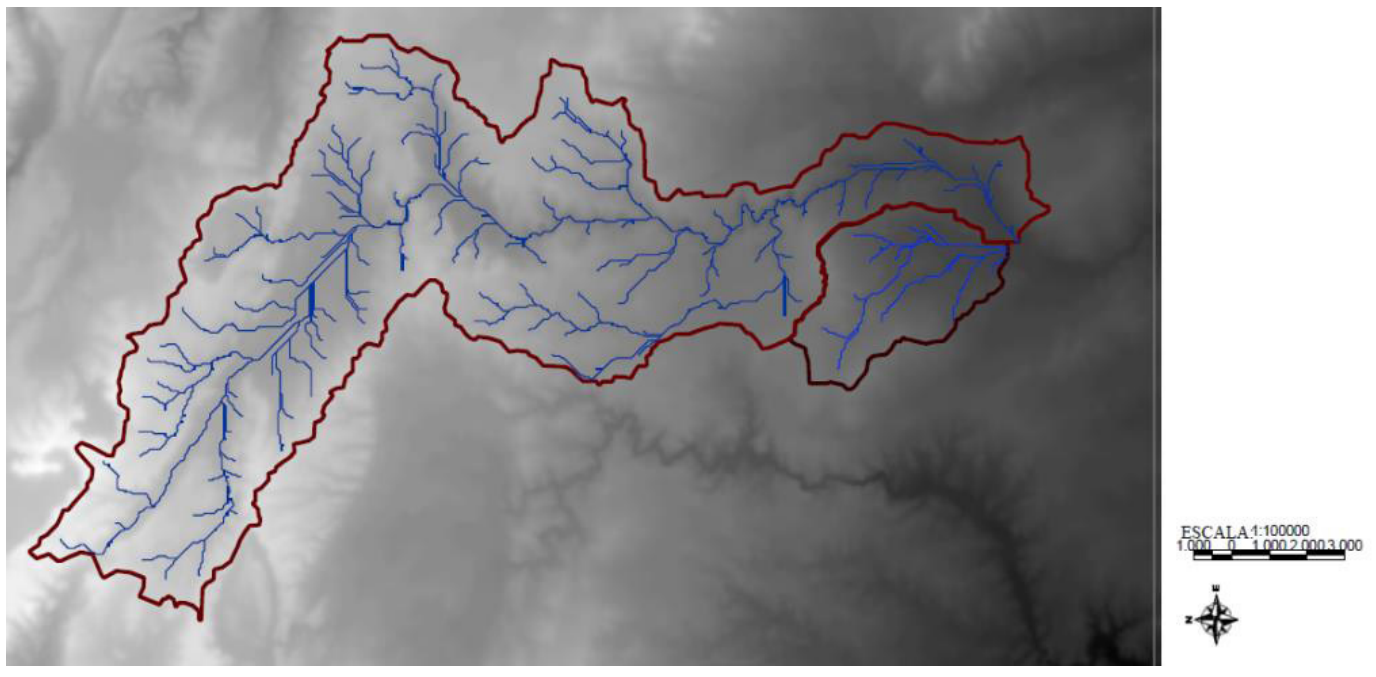

Respecto a los usos del suelo, la cuenca del Valdemembra se encuentra ocupada principalmente por masa forestal de repoblación o pinares resineros ocupando las zonas más abruptas y más altas. Las zonas llanas con materiales detríticos del terciario y los valles rellenos de aluviones están cultivados, ocupando una superficie muy importante, concentrándose en la Vega de Almodóvar y en el entorno de Motilla. El resto de usos del suelo son poco significativos, siendo la superficie artificial impermeable despreciable respecto a las otras. 
La otra cuenca afluente a Motilla, la del Riato, es más pequeña pero mucho más redondeada. La parte alta se asienta sobre relieves calcáreos cerca de Gabaldón, pasando hacia el sur a atravesar una franja estrecha de brechas rojas del Oligoceno, quedando la zona sur asentada sobre las arcillas y areniscas del Mioceno que caracterizan el entorno de Motilla. Es una cuenca sin caudal permanente y sin un cauce principal delimitado al estar totalmente cultivado. El cauce solo es apreciable dentro de la localidad a lo largo de la Avenida del Riato. Los usos del suelo son principalmente agrarios, ocupando la mayor parte de la cuenca baja. Hay zonas forestales de repoblación en los relieves de la parte alta. La zona más baja está ocupada por el casco urbano de Motilla, con un porcentaje destacable de la superficie total de la cuenca. Todo esto lleva a una cuenca con un coeficiente de escorrentía bastante alto, al presentar materiales bastante impermeables y cultivados, así como zonas totalmente urbanizadas, y por lo tanto impermeables de facto. La concentración de las zonas más impermeables en la zona baja y el hecho que en la época de mayor frecuencia de precipitaciones fuertes los cultivos de cereal estén cosechados y, por tanto, desprovistos de cobertura vegetal, favorece la torrencialidad de esta pequeña cuenca.

Un factor que se evidenció en las inundaciones de 2004 fue el efecto que los cambios en las costumbres de los habitantes tuvieron sobre la escorrentía. Las fuertes tormentas que se produjeron desde finales de agosto hasta el día 5 de septiembre generaron avenidas en todos los cursos de la zona, especialmente los día 3 y 4 de septiembre. Estas avenidas arrastraron la paja que estaba abandonada en los campos después de la cosecha. Hasta principios de este siglo la paja se recogía para alimentar el abundante ganado ovino y caprino de la zona. Cuando comenzó a crecer la burbuja inmobiliaria y la zona prosperó considerablemente se abandonaron muchas explotaciones ganaderas. La paja pasó de ser un recurso a un desecho y, al estar prohibida la quema de rastrojos en verano, se comenzó a abandonar en los campos. El resultado fue que la paja arrastrada por la escorrentía formó montoneras en todos los pasos de agua y puentes de la zona, cegándolos y formando balsas que acabaron por rebosar inundando muchos tramos de las carreteras, agravando la severidad de las inundaciones.

\subsubsection{Desarrollo del mapa de peligrosidad}

Para el estudio de la peligrosidad se realizó un estudio de las inundaciones históricas en la localidad, consultando documentación histórica, principalmente en hemerotecas, entrevistando a vecinos de la localidad y estudiando las adaptaciones estructurales de las viviendas del casco urbano. Combinando todos estos datos se realizaron dos mapas de peligrosidad histórica en el casco urbano de la localidad, uno para el río Valdemembra y otro para el Riato.

Los elementos tenidos en cuenta para la realización del mapa de peligrosidad histórica fueron todos aquellos elementos arquitectónicos que no tuviesen otro fin que el de elevar la vivienda respecto a la calle más allá de lo marcado por la ley o aquellos que permitiesen su aislamiento de la calle en caso de inundación. Estos incluyen los escalones y las rampas de acceso a los edificios (casas elevadas) y las guías para introducir tablas en caso de inundación colocadas en las puertas de las viviendas y de los comercios. Se realizó un estudio de campo en el que se señaló en un mapa de la localidad el tipo de adaptación: un símbolo tipo escalón $(\llcorner)$ para las viviendas con escaleras de entrada, un triángulo rectángulo $(\triangle)$ para las viviendas con rampa de acceso y un símbolo en forma de ' $h$ ' mayúscula $(\mathrm{H})$ para las viviendas con guías para poner tablas en las puertas. Además se pintó en color azul aquellos que tenían menos de $80 \mathrm{~cm}$ desde la calzada y en rojo aquellos que tenían más de $80 \mathrm{~cm}$ desde la calzada para relacionarlo después con la altura de la inundación y el grado de daño. La elección de este límite se debe a que $80 \mathrm{~cm}$ es aproximadamente la altura de una mesa, y está demostrado por la experiencia que los daños aumentan exponencialmente al alcanzar el agua esa altura debido a la presencia de los objetos más valiosos sobre las mesas.

A parte de las adaptaciones en la edificación, se tuvo en cuenta los datos de testigos, fotografías y prensa sobre inundaciones históricas así como las curvas de nivel de espaciado 0,5 metros obtenidas del MDT05. Estos datos ayudaron a completar las zonas con ausencia de información, a confirmar las alturas de agua que daban las adaptaciones en las distintas zonas y a explicar algunos desajustes observados. En la zona baja de la localidad hay adaptaciones de diferentes alturas muy próximas ente sí y sin delimitar una forma clara, lo que 
dificultaba el trazado de la zona inundable. Las curvas de nivel, los relatos de prensa y los testigos permitieron delimitar dos zonas deprimidas donde se alcanzaban alturas de agua mayores, quedando rodeadas por zonas con calados menores. Los mapas de peligrosidad obtenidos se pueden ver en las figuras 3 y 4.

Figura 3. Mapa de peligrosidad para el entorno del Riato.

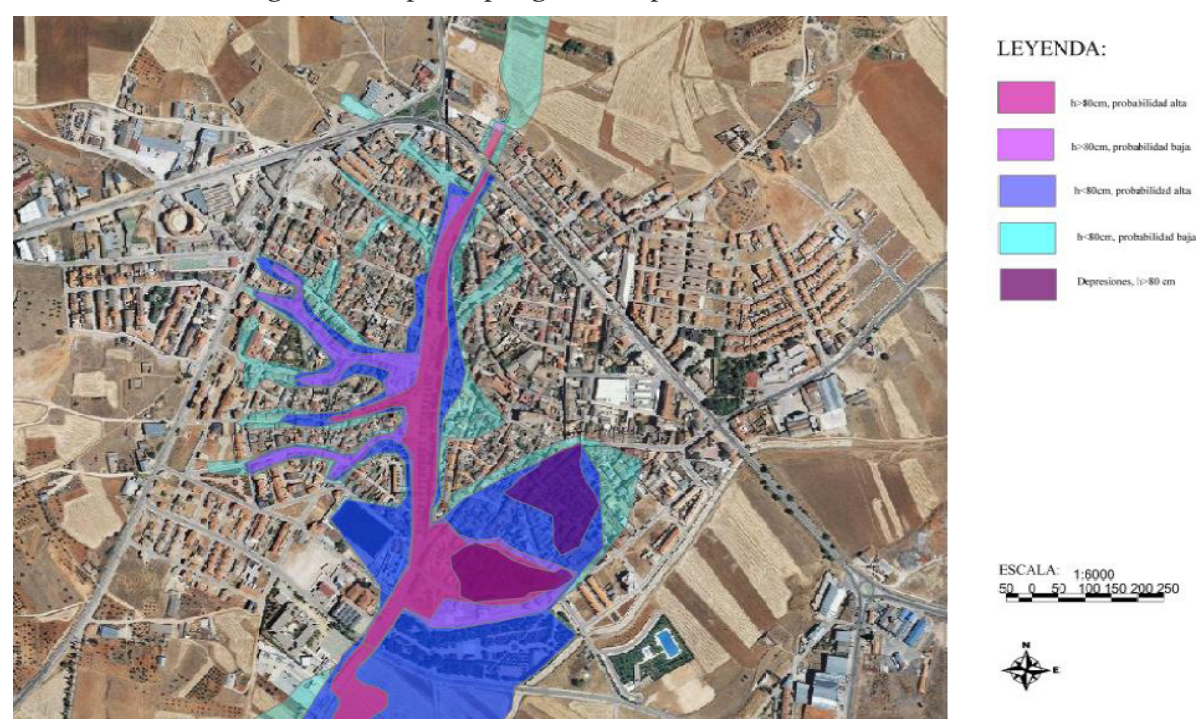

Los resultados de peligrosidad obtenidos cuadran con las características hidrológicas de las cuencas. En el caso del Riato, a pesar de ser una cuenca pequeña, presenta caudales punta importantes capaces de inundar amplias zonas de la localidad, lo que concuerda con la baja permeabilidad de la cuenca, especialmente en la parte baja, y la forma redondeada de ésta. En el caso del Valdemembra, la zona marcada como inundable concuerda con la propia geomorfología, coincidiendo prácticamente con la vega del río.

Esta situación se verá modificada probablemente en futuros eventos, al haberse ocupado la margen izquierda por donde solía 'salirse' el río, según los vecinos, con un polideportivo, con lo que los caudales desbordados se redirigirán hacia la margen derecha. Esto ocurre a la altura del puente que parte de la calle San Sebastián, que genera remansos y no permite desaguar correctamente el cauce. Por tanto, es probable que el agua se desborde hacia la zona baja de la localidad. Además, la presencia del muro de un parque en la zona baja en dirección perpendicular a la de la corriente propicia el embalsamiento del agua en esa misma zona como ya ocurrió en 2004.

Figura 4. Mapa de peligrosidad para el entorno del río Valdemembra.
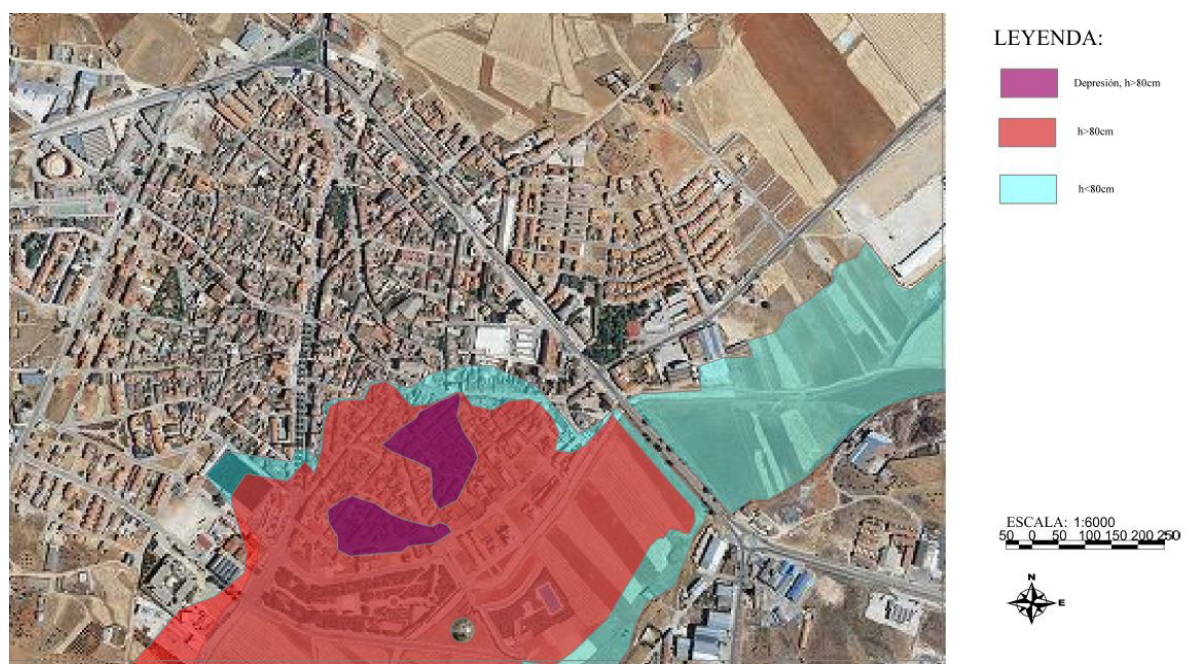


\subsubsection{Exposición}

La población expuesta al riesgo de inundación en Motilla ha sido difícil de calcular. La población total ronda los 6.200 habitantes. Sin embargo, no toda la población está expuesta, ya que las zonas altas de la localidad no se ven afectadas. La variación en las dimensiones de los diferentes tipos de viviendas por barrios ha hecho muy difícil un cálculo de la población afectada a partir del área inundada. Además recibe cada día un número muy importante de vecinos de poblaciones próximas que acuden a Motilla por diferentes razones (salud, comercio, transporte, justicia,...) al ser la capital de servicios de la comarca. Los lunes, cuando se instala el mercado en la Avenida del Riato, se da la mayor concentración de personas.

Además durante las fiestas patronales (principios de septiembre) se instala en la misma avenida la feria y la carpa del baile popular. Durante esos días, especialmente por las tarde y por las noches se concentra mucha población en la avenida, que es la zona más expuesta a las inundaciones. Estas instalaciones temporales, muy vulnerables a las inundaciones, y con altas ocupaciones temporales generan escenarios de alta exposición y alta vulnerabilidad que han sido causa de víctimas en el pasado. Un ejemplo reciente lo tenemos en la muerte de dos jubilados británicos en un mercadillo instalado en una rambla en la Cala de Finestrat (Alicante).

Así, los escenarios de mayor exposición son los barrios bajos de la localidad junto al río y la Avenida del Riato. En el primer caso es donde se han dado las inundaciones históricas más graves, estando la zona ocupada totalmente por viviendas, que se ven afectadas por las frecuentes avenidas que bajan por el Riato y por las menos frecuentes pero potencialmente más severas inundaciones procedentes del río. El peor escenario se daría de noche, cuando la mayor parte de la población está durmiendo en sus casas.

Por otro lado la Avenida del Riato presenta la mayor concentración de negocios de la zona, generando una alta exposición de bienes a las frecuentes inundaciones que bajan por dicha calle. Así mismo, las concentraciones de gente y de bienes que se dan durante la feria a principios de septiembre y durante el mercado todos los lunes son los escenarios que generan mayor exposición. La feria se instala en pleno Riato justo en los días en que las inundaciones son más habituales, lo que aumenta considerablemente la probabilidad de que se vea afectada, como ya ocurrió en 2004.

\subsubsection{Vulnerabilidad}

La vulnerabilidad en los barrios bajos se caracteriza por la presencia de una población envejecida habitando en viviendas de una sola planta de tapial o mampostería. Estas viviendas son muy vulnerables, ya que los ocupantes no pueden escapar al piso superior y se convierten en una trampa como se comprobó en Badajoz en 1997. Además la población mayor tiene menos capacidad de reacción, por lo que en sí misma es más vulnerable que otros colectivos.

Otro colectivo de alta vulnerabilidad son los inmigrantes. Al tener dificultades con el idioma pueden no comprender los mensajes de alerta. Además no conocen el clima local ni la historia por lo que probablemente no sepan que viven en una zona inundable y menos aún qué altura puede llegar a alcanzar el agua.

A parte de estos colectivos, la población presenta un bajo nivel formativo, con un nivel económico menor que la media nacional, lo que suele estar relacionado con una población más vulnerable frente a los riesgos. Además la percepción social del riesgo de inundación es nula, considerando la mayoría de los entrevistados que no hay riesgo potencial para sus vidas y escaso para los bienes, lo que agrava la vulnerabilidad.

Por último, cabe destacar las adaptaciones de muchas viviendas a las inundaciones, con pisos sobre elevados y ranuras para insertar tablas en las puertas, lo que reduce considerablemente la vulnerabilidad para bienes y personas, como también se pudo comprobar en 2004. 


\subsubsection{Riesgo}

A partir de las principales componentes del riesgo analizadas en los apartados anteriores se puede determinar un grado de riesgo para la localidad. En el caso del Riato el riesgo se concentra por un lado en la presencia en la avenida de la mayoría de los negocios lo que genera un escenario de daño económico importante. Por otro lado, las instalaciones temporales de alta vulnerabilidad y alta ocupación temporal durante la época más propicia a las inundaciones dentro del cauce del Riato genera un escenario de riesgo alto, tanto económico como para las personas. En la inundación de 2004 se registraron importantes pérdidas económicas en las instalaciones de la feria, pero afortunadamente no hubo daños personales importantes, aunque el suceso del mercadillo de Finestrat muestra el elevado riesgo para la vida de las personas de este tipo de instalaciones en zonas inundables.

En el caso del río, los barrios bajos de la localidad se pueden ver afectados por inundaciones con grandes calados, como en 1921. El hecho de que la población no recuerde esta inundación y no perciba el peligro agrava la vulnerabilidad, ya de por sí alta debida a las viviendas de una sola planta de tapial y ocupadas en muchos casos por una población envejecida. Aún no dándose calados altos la inundación de la zona baja genera daños económicos importantes al afectar a un gran número de viviendas. Además esto se verá agravado por la ocupación de la margen izquierda bloqueando y desviando la inundación hacia la otra margen en la que se encuentran las viviendas, agravándose el problema con un puente que limita la capacidad de desagüe del cauce.

\subsection{Cartografía oficial de riesgo de inundación}

El siguiente paso fue la recopilación de la documentación oficial referente a riesgo de inundación en la zona. Para ello se consultó el Sistema Nacional de Cartografía de Zonas Inundables (SNCZI) que recoge toda la cartografía oficial disponible sobre riesgo de inundación en el territorio nacional. En el caso del río Valdemembra en el entorno de Motilla del Palancar la cartografía recogida en el SNCZI se refiere a los tramos con estudios inventariados, a las zonas inundables (Zona Inundable con probabilidad baja: $\mathrm{T}=500$ años; $y$ Zonas Inundables con probabilidad media: $\mathrm{T}=100$ ) y a los caudales máximos esperables. La cartografía de tramos inventariados solo recoge aquellos tramos que presentan algún estudio, incluyendo el río Valdemembra a la altura de Motilla. La cartografía de zonas inundables la desarrolló la CHJ como base del programa LINDE. Los mapas de caudales máximos esperables los desarrolló el CEDEX a partir de datos de aforos en dichas cuencas. Así mismo, el cauce del Valdemembra está catalogado como área de riesgo potencial significativo de inundación pero aguas abajo de Motilla, a partir de El Peral hasta su confluencia con el Júcar.

Los dos mapas de zonas inundables recogidos en el SNCZI en Motilla son del río Valdemembra para periodos de retorno de 100 y 500 años. La cartografía de zonas inundables para baja frecuencia ( $T=500$ años) solo incluye un tramo de 3,24 kilómetros, desde el puente de la CM-2202 aguas arriba hasta el final del casco urbano. El método de cálculo hidrológico fue la aplicación HEC-1 y para el cálculo hidráulico se empleó HEC-2. El caudal utilizado para calcular la superficie inundada fue de $150 \mathrm{~m}^{3} / \mathrm{s}$ calculado a través de HEC-1. Esta aplicación del Cuerpo de Ingenieros del Ejército de Estados Unidos incorpora varias metodologías para la transformación de la lluvia en escorrentía. En este caso se utilizó el método del hidrograma unitario de Clark. Los datos de caudales fueron luego introducidos en HEC-2 para obtener la superficie teórica de inundación para ese caudal. La escala de la cartografía es 1:2000, se puede considerar una escala aceptable. Además se considera régimen alterado, es decir, que hay estructuras de laminación o de derivación de caudales que modifican el régimen natural. Por tanto, en la delimitación de la zona inundable no se tuvieron en cuenta, al menos en este tramo, datos históricos o geomorfológicos, sino solo los obtenidos a través de cálculos de ingeniería.

El caso de la cartografía de zonas inundables con frecuencia media ( $T=100$ años) se realizó de la misma forma. La diferencia es el caudal de cálculo que en este caso fue de $92 \mathrm{~m}^{3} / \mathrm{s}$. Como es de esperar, la superficie inundada es menor que la obtenida para un periodo de retorno de 500 años. 
El SNCZI se aprobó a partir de la Directiva 2007/60 sobre evaluación y gestión de riesgos de inundación de la Unión Europea. Sin embargo la región de Castilla-La Mancha no disponía de cartografía referente a inundaciones cuando entró en vigor dicha directiva. La región aprobó en 2010 el PRICAM (Plan Especial de Protección Civil ante el Riesgo de Inundaciones en Castilla-La Mancha). En él se recoge la cartografía regional creada sobre riesgo de inundación, encargada al CEDEX. Sin embargo, esta cartografía regional no es cuantitativa, sino que clasifica los núcleos poblacionales de la región en 5 niveles de riesgo en base a diferentes valores de exposición, vulnerabilidad y peligrosidad. No presenta una cartografía de la zona inundable, por lo que no podrá ser comparada con la obtenida en el presente trabajo. El nivel de riesgo de Motilla del Palancar en el PRICAM es de riesgo máximo (A1)

Por otro lado, se consultó con el ayuntamiento de Motilla, obteniendo como única documentación el PGOU de 1994 y un estudio hidrológico e hidráulico encargado por la CHJ como paso previo a la solución de los problemas de inundabilidad en la localidad. El PGOU no tiene en cuenta el riesgo de inundación, declarando como suelo urbanizable toda la vega del Valdemembra a excepción del propio cauce. El estudio encargado por la $\mathrm{CHJ}$ presenta cálculos hidrológico-hidráulicos para las tres cuencas afluentes al casco urbano de la localidad, separando la cuenca del Riato en dos subcuencas. Dicho trabajo, realizado en 2008, es un estudio hidrológico e hidráulico del río Valdemembra y las dos ramblas afluentes al Riato y un análisis de las posibles soluciones al problema de las inundaciones en el núcleo urbano de Motilla del Palancar. En dicho estudio se utilizó la aplicación MAXPLU del CEDEX para el cálculo de la distribución de la precipitación para diferentes periodos de retorno. En el estudio se incluye también un estudio de las precipitaciones máximas recogidas en los observatorios próximos a Motilla. Sin embargo se decidió tomar los valores obtenidos de la aplicación MAXPLU porque 'presentan órdenes de magnitud similares, permite un mejor análisis en la distribución espacial de la precipitación gracias al análisis regional que se llevó a cabo y porque su utilización se encuentra ampliamente sancionada por la práctica a nivel nacional'. Esta justificación presenta un problema, ya que los valores obtenidos suelen ser inferiores a los valores reales. Así, según los datos tomados para el estudio hidrológico a partir de MAXPLU la precipitación máxima diaria para un periodo de retorno de 500 años para cualquier celda en las que se dividió la cuenca nunca superaría los $140 \mathrm{~mm}$. Sin embargo, en el mismo estudio se han calculado las precipitaciones a partir de los datos reales de precipitación, utilizando un ajuste SQRT, obteniéndose valores de más $140 \mathrm{~mm}$ diarios para T=100 para la estación de Almodóvar del Pinar, llegando casi a $200 \mathrm{~mm}$ diarios para un periodo de retorno de 500 años. Es cierto que los datos de precipitaciones reales son escasos y los registros tienen poca extensión temporal, lo que limita su aplicación.

En el caso de la estación del SAIH de Almodóvar, los registros comenzaron en junio de 1989 y hay datos hasta 2008 (fecha de realización del estudio). En ese periodo de casi 20 años se superaron los $110 \mathrm{~mm}$ diarios en dos ocasiones (1997 y 2004), mientras que con los datos de MAXPLU esos valores no se superarían en ninguna celda de la cuenca salvo para periodos de retorno mayores de 200 años.

A continuación se calculó con esas intensidades diarias a partir de las curvas intensidad-duración (para cada celda del modelo) la intensidad máxima esperable según el método propuesto por la Instrucción de Carreteras 5.2-IC y los hietogramas sintéticos según el método de los bloques alternos, tomando como duración de la tormenta 24 horas, según los autores, por la falta de datos. Esto presenta otro problema, ya que las mayores precipitaciones también son las más intensas y se concentran en pocas horas, como en 1921 y 2004.

A partir de dichos datos, utilizando el modelo TOPKAPI se calculó el caudal. Este modelo es bastante complejo. Es un modelo distribuido (se pueden calcular caudales en cualquier celda) y tiene en cuenta la escorrentía superficial y la subsuperficial, además de delimitar las áreas contribuyentes variables en función de la saturación del suelo. En la simulación se tuvieron en cuenta tres escenarios: con el suelo seco, medio y húmedo.

Los caudales obtenidos en este estudio para periodos de retorno de 2, 5, 10, 50, 100, 200 y 500 años se recogen en la tabla 2. 
Los caudales máximos obtenidos para el periodo de retorno de 500 años son inferiores a $50 \mathrm{~m}^{3} / \mathrm{s}$, siendo muy pequeña la variación en función del estado de humedad del suelo. Comparándolos con los valores del resto de métodos recogidos en el SNCZI se puede ver que es el doble del caudal del mapa de caudales máximos para ese periodo de retorno, pero sigue siendo un tercio del recogido en la cartografía de zonas inundables. Para periodos menores se puede apreciar que los valores son conservadores, quedando del lado de la inseguridad. Para un periodo de retorno de 100 años da caudales inferiores a $20 \mathrm{~m}^{3} / \mathrm{s}$, mientras que el dato utilizado en la cartografía de zonas inundables es de $92 \mathrm{~m}^{3} / \mathrm{s}$, más de cuatro veces mayor. La diferencia es sustancial, ya que el propio estudio calcula una capacidad de desagüe del cauce del Valdemembra de entre 40 y $45 \mathrm{~m}^{3} / \mathrm{s}$, con lo que según sus cálculos, estaría diseñado prácticamente para caudales con periodos de retorno de 500 años. Sin embargo, si se toma los caudales utilizados en la cartografía de zonas inundables, no sería suficiente para caudales con periodos de retorno de 100 años, además siendo superado de una forma amplia.

Tabla 2. Caudales punta para diferentes periodos de retorno y diferentes grados de humedad del suelo para el río Valdemembra.

\begin{tabular}{|c|c|c|c|c|c|c|c|c|}
\hline & \multicolumn{9}{|c|}{ Q pico (m/s) } \\
\hline Periodo de retorno (años) & T2 & T5 & T10 & T25 & T50 & T100 & T200 & T500 \\
\hline Valdemembra-Seco & 0,103 & 0,103 & 0,103 & 0,103 & 1,55 & 7,5 & 20,65 & 45,31 \\
\hline Vademembra-Medio & 0,103 & 0,291 & 1,431 & 5,424 & 10,58 & 17,5 & 27,17 & 49,2 \\
\hline Valdemembra-Húmedo & 0,103 & 0,759 & 2,179 & 5,928 & 10,72 & 17,5 & 27,38 & 49,2 \\
\hline
\end{tabular}

El estudio también hace un análisis de la capacidad de los pasos superiores sobre el cauce. Determina que el puente de la N-III es suficiente y no provoca remansos, mientras que el puente que sale de la calle San Sebastián no es suficiente, generando un remanso importante que limita la capacidad de desagüe del cauce. El puente de la Ronda Sur no se considera limitante porque el factor limitador es la cota de la orilla izquierda, aunque también provoca remansos.

Como se ha visto, se dispone de una cartografía considerable sobre riesgo de inundación en la zona, pero muy variable en cuanto a los valores de caudales. Estas variaciones, permiten poner en duda los datos aportados por estas cartografías.

\subsection{Comparación de la cartografía obtenida con la cartografía oficial}

Si comparamos la cartografía obtenida con la disponible en SNCZI, podemos ver que la zona marcada en este último como inundable entorno al río Valdemembra para periodos de retorno de 100 y 500 años (figuras 5 y 6) no incluye la zona que siempre se inunda en la localidad correspondiente a la parte baja del casco urbano (figura 7). Estas zonas son las que se han inundado en todas las inundaciones, y las que presentan la mayor concentración de adaptaciones en la construcción y se corresponden con las dos zonas deprimidas localizadas con las curvas de nivel. Este error en la cartografía del SNCZI permite intuir una falta de precisión en los cálculos o la falta de consideración de otras informaciones a parte de los propios cálculos informáticos. Además, estas zonas deprimidas presentan un claro peligro para la vida de las personas, ya que fue donde se concentraron las víctimas en el evento de 1921, alcanzando el agua hasta 2 metros en el interior de algunas viviendas.

El resto de estudios y cartografías presentan valores de caudales esperables mucho menores para los mismos periodos de retorno, lo que aún es más grave. Por tanto su imprecisión es aún mayor y quedan totalmente del lado de la inseguridad.

Especialmente grave es el caso del estudio que posee el consistorio ya que para periodos de retorno de 500 años da caudales para el río Valdemembra de $45 \mathrm{~m}^{3} / \mathrm{s}$, mientras que la cartografía del SNCZI se realizó para caudales de $150 \mathrm{~m}^{3} / \mathrm{s}$. Según dicho estudio el encauzamiento del río sería suficiente incluso 
para periodos de retorno de 500 años con caudales de no más de $50 \mathrm{~m}^{3} / \mathrm{s}$, mientras que en el SNCZI supera los $150 \mathrm{~m}^{3} / \mathrm{s}$, tres veces mayor.

Figuras 5 y 6. Cartografías de la zona inundable para $\mathrm{T}=100$ y $\mathrm{T}=500$ años recogida en el SNCZI para el entorno de Motilla del Palancar.
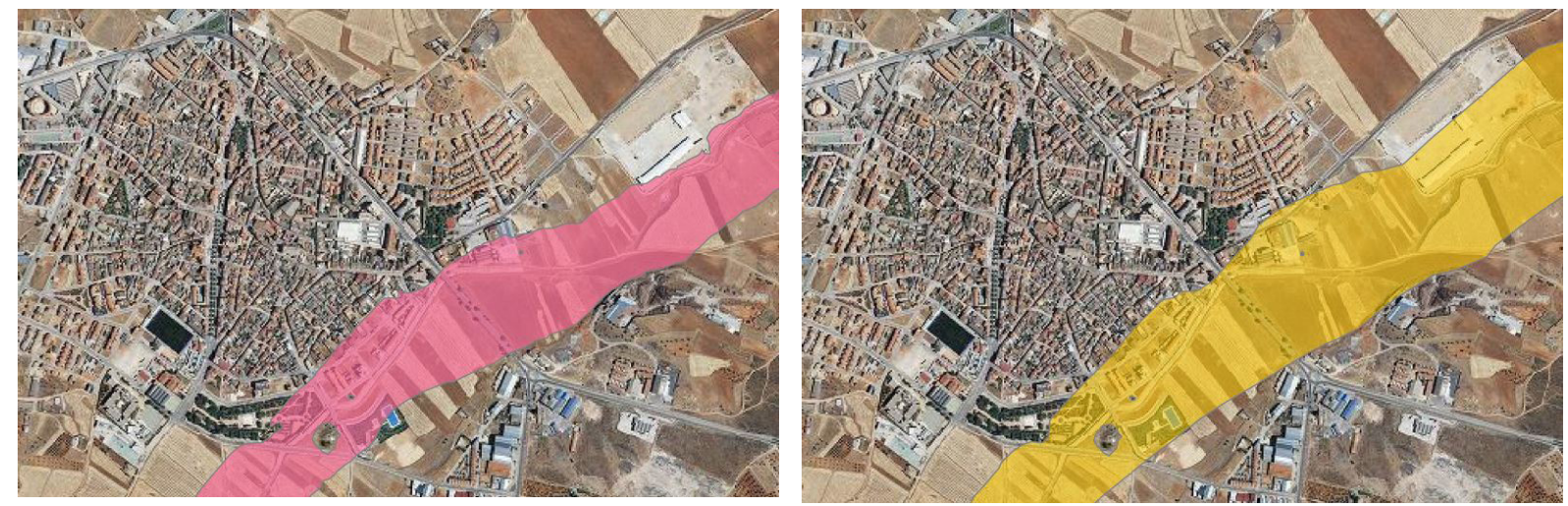

Figura 7: Cartografía de peligrosidad obtenida en el presente estudio para el río Valdemembra.

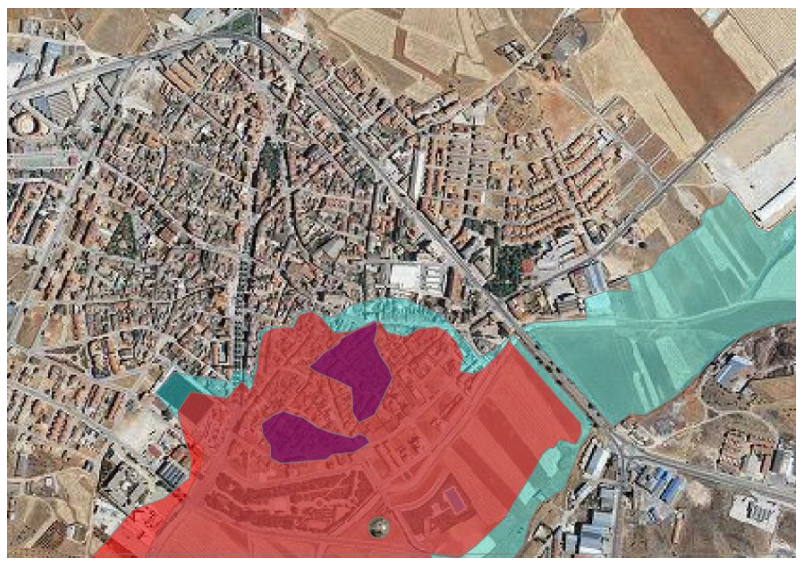

Así mismo el PGOU no tiene en cuenta el riesgo de ninguna forma. Por tanto, el riesgo sigue aumentando al continuar la urbanización de la vega del río, declarada como urbanizable. La vega cada vez está más ocupada y ya se ha empezado a urbanizar la margen izquierda. En ese lado se ha construido un polideportivo que ocupa todo el ancho de la llanura de inundación con un terraplén de más de 1 metro que corta el desagüe de las aguas desbordadas. Según los vecinos el río tendía a salirse por esa margen. Por tanto en futuras inundaciones el agua no podrá desaguar a través de la margen izquierda, desbordándose hacia la margen derecha en dirección al casco urbano, aumentando la peligrosidad. Tampoco hay ninguna norma de urbanismo que obligue a una construcción adaptada a las inundaciones como se venía haciendo tradicionalmente, de tal forma que junto al mismo cauce hay viviendas de construcción reciente con garaje en el sótano o el piso a nivel de calle.

Respecto al cauce del Riato solo el estudio que posee el ayuntamiento realiza cálculos de caudales. Los caudales obtenidos son ínfimos, con $1 \mathrm{~m}^{3} / \mathrm{s}$ para un periodo de retorno de 10 años, $4 \mathrm{~m}^{3} / \mathrm{s}$ para 50 años y $19 \mathrm{~m}^{3} / \mathrm{s}$ para 500 años. Los caudales para periodos cortos no se corresponden con lo observado. Los testigos y los datos muestran que cada vez que llueve con intensidad la rambla baja con agua, teniendo inundaciones importantes cada 10 años aproximadamente, con caudales a todas luces superiores a $5 \mathrm{~m}^{3} / \mathrm{s}$.

\section{CONCLUSIONES}

La localidad de Motilla del Palancar se encuentra expuesta a un riesgo derivado de fenómenos de inundación originados principalmente por lluvias intensas relacionadas con sistemas convectivos 
generados en verano, principalmente a finales de la estación. La población presenta una peligrosidad considerable. Por un lado, la rambla que discurre por la Avenida del Riato genera inundaciones con frecuencia alta. Estas inundaciones discurren por la calle principal de la ciudad afectando a un gran número de establecimientos comerciales generando principalmente daños económicos. También supone un peligro para las personas que pueden ser arrastradas por la corriente. Especialmente vulnerables son las instalaciones temporales como el mercado o la feria, que presentan altas concentraciones de gente y un gran número de bienes. Además inunda la zona baja del casco urbano, afectando a un gran número de viviendas. Por otro lado, las inundaciones debidas al río son menos frecuentes, pero la altura del agua es mayor. Prueba de ello es la inundación de 1921. Afectan a la zona baja de la localidad, donde vive un gran número de personas mayores en viviendas antiguas de tapial y de una sola planta, muy vulnerables.

La situación se ha agravado debido a la acción humana, al invadirse zonas inundables en la llanura de inundación del río, aumentando la exposición de bienes y personas. Además, la construcción de instalaciones en la margan izquierda ha eliminado la vía de desagüe natural que tenía el río por esa zona. En un futuro evento de gran intensidad, el cauce actual no sería suficiente y el puente de la calle San Sebastián provocaría un embalsamiento que, no pudiendo desbordar hacia la margen izquierda como venía haciendo hasta ahora, lo hará hacia la margen derecha, inundando la localidad. Sumado al efecto barrera del muro del parque y a que la zona baja de la ciudad está deprimida respecto al cauce, provocará alturas del agua que pueden suponer un peligro para la vida de un gran número de personas. El tipo de construcción que se vería afectado, muy vulnerable, y la población de avanzada edad que las habita originan un escenario de riesgo alto para la vida de las personas.

Ante esta situación de riesgo las autoridades locales no han realizado ninguna acción. Solo se ha realizado un estudio por parte de la DHJ sobre este tema. Este estudio, sin embargo, da valores de caudal mucho menores que los reales, por lo que queda del lado de la inseguridad, privando a los organismo locales del conocimiento del riesgo real, transmitiendo una falsa sensación de seguridad. El riesgo no ha sido tenido en cuenta en la ordenación territorial, lo que ha agravado el riesgo. Sí hay una cartografía de peligrosidad desarrollada en el SNCZI, con zonas inundables cartografiadas para periodos de retorno de 100 y 500 años, aunque no incluye las zonas habitualmente inundadas del casco urbano, por lo que su precisión es cuestionable. Por otro lado se dispone de un sistema de gestión de emergencias eficaz, lo que ayudará a minimizar los efectos de un desastre, y dos planes autonómicos, el METEOCAM y el PRICAM.

Por tanto, la conclusión del presente trabajo es que le grado de riesgo en Motilla es importante, tanto por la peligrosidad, como por la vulnerabilidad y la exposición. Además ha aumentado en los últimos años y seguirá aumentando si se sigue urbanizando en zonas inundables y no se corrigen las situaciones actuales al aumentar la exposición y agravarse la peligrosidad. Se recomienda realizar las siguientes actuaciones:

- Aprobación definitiva del POM, teniendo en cuenta el riesgo de inundación y limitando la edificación en zonas inundables, o como mínimo condicionándola.

- Realización de campañas de información y concienciación de la población.

- Desarrollo y aprobación de Plan de Actuación en el Ámbito Local, derivado del PRICAM.

- Soterramiento de la rambla bajo la Avenida del Riato.

- Aumento de la sección del encauzamiento del río Valdemembra a su paso por la localidad, sustituyendo los puentes con problemas de drenaje por otros con mayor luz.

\section{BIBLIOGRAFÍA}

ABC 22-09-1921, Madrid. Hemeroteca Digital del Diario ABC.

ABC 23-09-1921, Madrid. Hemeroteca Digital del Diario ABC.

ABC 03-01-1997, Madrid. Hemeroteca Digital del Diario ABC. 
AGENCIA DEL AGUA DE CASTILLA-LA MANCHA, (2012): Acuiferos, [web], <http://pagina.jccm.es/ agenciadelagua/index.php?id=24\&p=11>

ARENAS, M. et al., (1993): 'Nuevos datos sobre la crecida del Júcar de octubre de 1982'. Revista de Obras Públicas $\mathrm{N}^{\circ}$ 3.323. Año 140. Julio-Agosto.

ÁVILA, J. V., EL DIGITAL DE CUENCA, (1-10-2011): [Hemeroteca Digital],http://eldiadigital.es/ not/33559/noventa anos de la inundacion que vistio de luto y lodo a motilla/

AYALA-CARCEDO, F. J., OLCINA CANTOS, J. (2002): 'Riesgos Naturales', Ariel Ciencia.

DE LAS HERAS, J., EL PAÍS, (3-07-1979): [Hemeroteca digital], http://elpais.com/diario/1979/07/03/ espana/299800806_850215.html

DEMARCACIÓN HIDROGRÁFICA DEL JÚCAR, Agua/La cuenca hidrográfica/Medio físico, [web], (2012): http://www.chj.es/es-es/medioambiente/cuencahidrografica/Paginas/MarcoFisico.aspx

FICHA MUNICIPAL DE MOTILLA DEL PALANCAR, Instituto de Estadística de Castilla-La Mancha.

GARCÍA-DIEGO J. A. et al.,(1983): 'Estudio conjunto sobre la presa romana de Consuegra.' Revista de Obras Públicas, agosto 1983. No 3216, pp. 585-599.

GARCÍA MARCHANTE, J. S. et al., (2001): 'La empresarialidad rural en el marco del desarrollo territorial. Un estudio de caso'. Boletín de la AGE No 43, pp. 107-129. Facultad de Ciencias de la Educación y Humanidades. Universidad de Castilla-La Mancha.

GIL OlCINA, A.; MORAlES GIL, A. (ed.) (1989): 'Avenidas fluviales e inundaciones en la cuenca del Mediterráneo', Alicante, Instituto universitario de Geografía y CAM, 585 págs.

GIL OLCINA, A. y OLCINA CANTOS, J. (1997): 'Climatología General', Barcelona. Edit. Ariel.

GIL OLCINA, A.; MORALES GIL, A.(Ed.) (2011): 'Causas y consecuencias de las sequías en España', Alicante, Instituto Universitario de Geografía y CAM, 574 págs.

GIL OLCINA, A., OLCINA CANTOS, J. y RICO AMORÓS, A. M. (Edits.) (2004): 'Aguaceros, aguaduchose inundaciones en áreas urbanas alicantinas'. Alicante, Publicaciones de la Universidad de Alicante.

INDICADORES SOCIALES 2011. EDUCACIÓN. <http://www.ine.es/daco/daco42/sociales11/sociales.htm>

MAGNA Motilla del Palancar, Hoja 691 (24-27), Escala 1:50.000, Instituto Geológico y Minero de España, 1976.

MAGNA Valera de Abajo, Hoja 663 (24-26), Escala 1:50.000, Instituto Geológico y Minero de España, 1976.

MARTÍN VIDE, J. y OLCINA CANTOS, J. (2001): 'Climas y tiempos de España', Madrid, Alianza Editorial.

Memoria total del PRICAM (Plan de Protección Civil ante el Riesgo de Inundaciones en Castilla-La Mancha, 2010. Junta de Comunidades de Castilla-La Mancha).

OlCINA, J. (1994): 'Riesgos climáticos en la Península Ibérica', Madrid, Libros Penthalon, 440 págs.

OLCINA, J. (1994): 'Tormentas y granizadas en las tierras alicantinas', Alicante, Instituto Universitario de Geografía, 317 págs.

OLCINA CANTOS, J. (1995): 'El factor climático y la ordenación territorial. Los riesgos climáticos' en 'Situaciones de riesgo climático en España' (Creus Novau, J. edit.), Asociación de Geógrafos Españoles (Grupo de Climatología) e Instituto Pirináico de Ecología, Jaca, pp. 15-69.

OLCINA CANTOS, J. (2004): 'Riesgo de inundaciones y ordenación del territorio en la escala local. El papel del planeamiento urbano municipal', Boletín de la Asociación de Geógrafos Españoles, $\mathrm{n}^{\circ} 37$ (monográfico "Agua y Ciudad"), Madrid, Asociación de Geógrafos Españoles, pp. 49-84.

OLCINA CANTOS, J. (2004): 'Riesgos fluviales y ordenación del territorio', en 'Alteración de los regímenes fluviales peninsulares' (Gil Olcina, A. coord..), Murcia, Fundación CajaMurcia, pp.45-97.

OLCINA CANTOS, J. (2006): ¿Riesgos Naturales? I. Sequías e inundaciones. Editorial DaVinci Continental. Colección Geoambiente XXI. Barcelona, 220 pp. 
Registro del aforo de Mahora, ${ }^{\circ}$ de estación 8085, periodo 1916-1948. Demarcación Hidrográfica del Júcar. Ministerio de Agricultura, Alimentación y Medio Ambiente.

RIESCO, J., ALCOVER, V. 'Algunas consideraciones sobre lluvias intensas en el Mediterráneo Occidental: Revisión de un episodio en la Comunidad Valenciana.' GPV del CMT en Valencia, INM.

SAUQUILlO, A., Grupo de Investigación de Hidrogeología, UPV, Real Academia de Ciencias, 'Relaciones río acuífero en la Mancha Oriental'.

Visor web del Sistema Nacional de Cartografía de Zonas Inundables (2012): Ministerio de Agricultura, Alimentación y Medio Ambiente, Gobierno de España.

WETTERZENTRALE, Kartenarchiv ab 1871 [Archivos de mapas desde 1871], (2012), http://www. wetterzentrale.de/topkarten/fsreaeur.html

\section{RECURSOS ELÉCTRÓNICOS}

http://foro.tiempo.com/especial-tormentas-de-verano-septiembre-este-y-baleares-t14665.396.html http://lovemotilla.blogspot.com/

http://villadtembleque.blogspot.com.es/2010/01/la-inundacion-de-consuegra-de-1891-2.html http://www.tiempo.com/ram/1674/vrtice-mesoescalar-de-niveles-medios-de-origen-convectivo/ http://www.tiemposevero.es/ver-reportaje.php?id=56 